\title{
BMJ Open Drugs and life-threatening ventricular arrhythmia risk: results from the DARE study cohort
}

\author{
Abigail L Coughtrie, ${ }^{1,2}$ Elijah R Behr, ${ }^{3,4}$ Deborah Layton, ${ }^{1,2}$ Vanessa Marshall, ${ }^{1}$ \\ A John Camm, ${ }^{3,4,5}$ Saad A W Shakir ${ }^{1,2}$
}

To cite: Coughtrie AL, Behr ER, Layton D, et al. Drugs and life-threatening ventricular arrhythmia risk: results from the DARE study cohort. BMJ Open 2017;7:e016627. doi:10.1136/ bmjopen-2017-016627

- Prepublication history for this paper is available online To view these files please visit the journal online (http://dx.doi. org/10.1136/bmjopen-2017016627).

Received 20 March 2017 Revised 5 July 2017 Accepted 10 August 2017
CrossMark

${ }^{1}$ Research Department, Drug Safety Research Unit, Southampton, UK

${ }^{2}$ School of Pharmacy and Biomedical Science, University of Portsmouth, Portsmouth, UK ${ }^{3}$ Cardiology Clinical Academic Group, St George's University of London, London, UK

${ }^{4}$ Cardiology Clinical Academic Group, St George's University Hospitals NHS Foundation Trust, London, UK

${ }^{5}$ Faculty of Medicine, Imperial College London, London, UK

Correspondence to

Dr Abigail L Coughtrie; abigail.coughtrie@dsru.org

\section{ABSTRACT}

Objectives To establish a unique sample of proarrhythmia cases, determine the characteristics of cases and estimate the contribution of individual drugs to the incidence of proarrhythmia within these cases.

Setting Suspected proarrhythmia cases were referred by cardiologists across England between 2003 and 2011. Information on demography, symptoms, prior medical and drug histories and data from hospital notes were collected. Participants Two expert cardiologists reviewed data for 293 referred cases: 130 were included. Inclusion criteria were new onset or exacerbation of pre-existing ventricular arrhythmias, QTc $>500 \mathrm{~ms}$, QTc $>450 \mathrm{~ms}$ (men) or $>470 \mathrm{~ms}$ (women) with cardiac syncope, all secondary to drug administration. Exclusion criteria were acute ischaemia and ischaemic polymorphic ventricular tachycardia at presentation, structural heart disease, consent withdrawn or deceased prior to study. Descriptive analysis of Caucasian cases (95\% of included cases, $\mathrm{n}=124$ ) and culpable drug exposures was performed. Results Of the 124 Caucasian cases, 95 (77\%) were QTC interval prolongation-related; mean age was 62 years (SD $15)$, and $63 \%$ were female. Cardiovascular comorbidities included hypertension (53\%) and patient-reported 'heart rhythm problems' (73\%). Family history of sudden death (36\%) and hypokalaemia at presentation (27\%) were common. 165 culpable drug exposures were reported, including antiarrhythmics (42\%), of which amiodarone and flecainide were the most common. Sotalol, a beta-blocking agent with antiarrhythmic activity, was also common (15\%). $26 \%$ reported multiple drugs, of which $84 \%$ reported at least one cytochrome (CYP) P450 inhibitor. Potential pharmacodynamics interactions identified were mainly QT prolongation (59\%).

Conclusions Antiarrhythmics, non-cardiac drugs and drug combinations were found to be culpable in a large cohort of 124 clinically validated proarrhythmia cases. Potential clinical factors that may warn the prescriber of potential proarrhythmia include older women, underlying cardiovascular comorbidity, family history of sudden death and hypokalaemia.

\section{INTRODUCTION}

Drug-induced arrhythmia, or proarrhythmia, is the induction or exacerbation of cardiac arrhythmia associated with administration of a drug. The majority of drug-induced

\section{Strengths and limitations of this study}

- The Drug-induced Arrhythmia Risk Evaluation study has allowed the development of a cohort of cases of proarrhythmia.

- These cases have provided crucial safety information, as well as underlying clinical and genetic data.

- Only patients who did not die as a result of the proarrhythmia could be included.

- Referral of cases by cardiologists alone may have led to the underestimation of the prevalence of druginduced arrhythmia from non-cardiac drugs.

- The analysis of ethnicity and differences in risk of QT prolongation could not be investigated.

arrhythmic events relate to marked prolongation of the QT interval of the ECG, which can lead to the distinctive polymorphic ventricular tachycardia (VT) and 'Torsades de Pointes' (TdP), which in turn may lead to ventricular fibrillation (VF) and sudden death. ${ }^{1}$ This is also known as the acquired long QT syndrome (aLQTS). Occasionally polymorphic and monomorphic VT without QT prolongation can occur. ${ }^{23}$ In addition to drugs, other causes of aLQTS include endocrine disorders, ${ }^{4}$ cirrhosis, ${ }^{5}$ HIV and AIDS, ${ }^{6}$ inflammation and immunity, ${ }^{7}$ autoimmune disease, ${ }^{8}$ structural heart disease, ${ }^{9}$ electrolyte imbalances ${ }^{10}$ and eating disorders. ${ }^{11}$

Drug-induced arrhythmia is associated with the use of cardiovascular agents (particularly class III antiarrhythmic drugs) and also with many non-cardiovascular indicated drugs within different therapeutic categories, including antihistamines, antipsychotics and antimicrobials; an up-to-date list is maintained on the CredibleMeds register (Azcert, https://crediblemeds.org/). Currently, there is substantial evidence to support a clear association between over 50 different drugs and risk of $\mathrm{TdP}$, even when taken according to the terms of the 
marketing authorisation, with a number of these being withdrawn from the market. ${ }^{12-14}$ Mechanistic proposals for clinical features such as electrolyte imbalance include block of the rapid form of the delayed rectifier potassium current $\left(\mathrm{I}_{\mathrm{Kr}}\right)$ in cardiomyocytes ${ }^{15-17}$ Genetic factors have also been identified. These include single nucleotide polymorphisms in the NOS1AP gene encoding the nitric oxide synthase 1 adaptor protein ${ }^{18}$; and mutations in potassium channel genes KCNH2, KCNQ1, KCNE1 and KCNE2 and/or the sodium channel gene SCN5A. ${ }^{19} 20$ Such mutations are also recognised to cause congenital long QT syndrome (cLQTS). ${ }^{21}$ Other notable risk factors include female sex, bradycardia, recent cardioversion, pre-existing electrolyte disturbance, elevated plasma concentrations and/or rapid infusion of QT-prolonging drugs and digitalis toxicity. ${ }^{22-24}$

Following the removal of several QT-prolonging drugs because of associated sudden deaths, ${ }^{25-28}$ risk minimisation strategies were introduced to mitigate the arrhythmic risk posed by drugs, including clinical studies to assess the proarrhythmic potential for a new drug within the premarketing development programme. ${ }^{29}$ However it has been recognised that there remain limitations in the conduct of clinical studies designed to evaluate a drug's potential for QT prolongation and applicability of results to vulnerable patients. ${ }^{30}$ Because of the unpredictable nature of the condition, the Drug-induced Arrhythmia Risk Evaluation (DARE) study aimed to improve the understanding of the epidemiology of proarrhythmia by establishing a cohort of cases of drug-induced arrhythmia reported throughout England, to characterise typical patients with proarrhythmia and to describe the drugs found to be culpable in these cases of proarrhythmia. This manuscript is a per-protocol descriptive analysis of risk factors for the condition and the contribution of individual drugs to the risk of drug-induced arrhythmic events.

\section{METHODS}

\section{Study design and setting}

Cardiologists across England were notified of the study by the British Pacing and Electrophysiology Group and the British Cardiac Society and asked to recruit patients. Study awareness and participation was further promoted by project presentation and local interaction across the country. Cases of suspected proarrhythmia were referred by cardiologists in England between March 2003 and July 2011. All consenting cases attended a face-to-face interview with a regional study nurse (North, South or Midlands regions) between May 2005 and August 2011.

\section{Participants}

Cases of proarrhythmia were included if they had one or more of the following criteria, all diagnosed as secondary to therapeutic drug administration or overdose: documented TdP, VF or VT (polymorphic or monomorphic, not associated with QT prolongation); exacerbation of pre-existing non-sustained arrhythmias to sustained; severe prolongation of the QTc interval corrected using Bazett's formula ( $>500 \mathrm{~ms}$ ) without symptoms; or moderate prolongation of the QTc interval ( $\geq 450 \mathrm{~ms}$ in men or $\geq 470 \mathrm{~ms}$ in women) with a clinical history of cardiac syncope.

All cases were reviewed by at least two experienced cardiologists, using hospital notes and interview questionnaire information, to ensure appropriate inclusion of cases. Patients with acute ischaemia, ischaemic polymorphic VT and structural heart disease (using symptoms, history of ischaemia and associated therapy, risk factors or ECG, stress test and coronary angiography results) were excluded. Case presentation (asymptomatic, syncope, VT, $\mathrm{VF}$ and/or TdP) and aetiology (QT prolongation-associated and non-QT prolongation-associated) were ascertained. Drugs received by the patient were adjudicated for culpability in contributing to proarrhythmia according to the clinical data, timing of medication and the presenting event that prompted referral. Prior reports of association with proarrhythmia were also taken into account, although drugs thought to contribute to causation but without such data were not excluded.

No sample size calculation could be performed for the study as, at the time of study initiation, the natural history, relative risk and potential risk factors of proarrhythmia were largely unknown.

\section{Variables}

A proforma questionnaire obtained patients' self-reported information on age, gender, ethnicity, weight, height, smoking status, alcohol consumption, symptoms before, during and after the event (including 'blackout', 'near blackout', 'dizziness/light-headedness' and 'palpitations'), medication taken before, during and after the event (including prescription, over-the-counter/herbal and recreational), any medical and cardiovascular history (including angina, myocardial infarction, 'heart failure', 'heart valve problem', 'heart rhythm problem', 'high blood pressure', hypokalaemia, hypothyroidism, diabetes mellitus, 'stroke', transient ischaemic attack, 'liver problem', 'kidney problem') and family medical history (including 'sudden death' and 'unexplained blackout'). History of proarrhythmic events was validated using each patient's hospital notes and an ECG taken at the time of the interview. Patient hospital notes, where available, were also used to validate drug history (including the $\operatorname{drug}(\mathrm{s})$ considered to be related to the proarrhythmic event), and medical and cardiovascular history for all cases.

Culpable drugs were mapped to the Anatomical Therapeutic Chemical classification system. The drugs were then classified according to the CredibleMeds register risk of causing QT prolongation and/or TdP into the following groups: known risk, possible risk, conditional risk and no known risk. Drugs are classified as having known risk when there is substantial evidence for QT interval prolongation and TdP risk when used according to the label; possible risk when there is substantial evidence for QT interval prolongation but insufficient evidence of TdP 


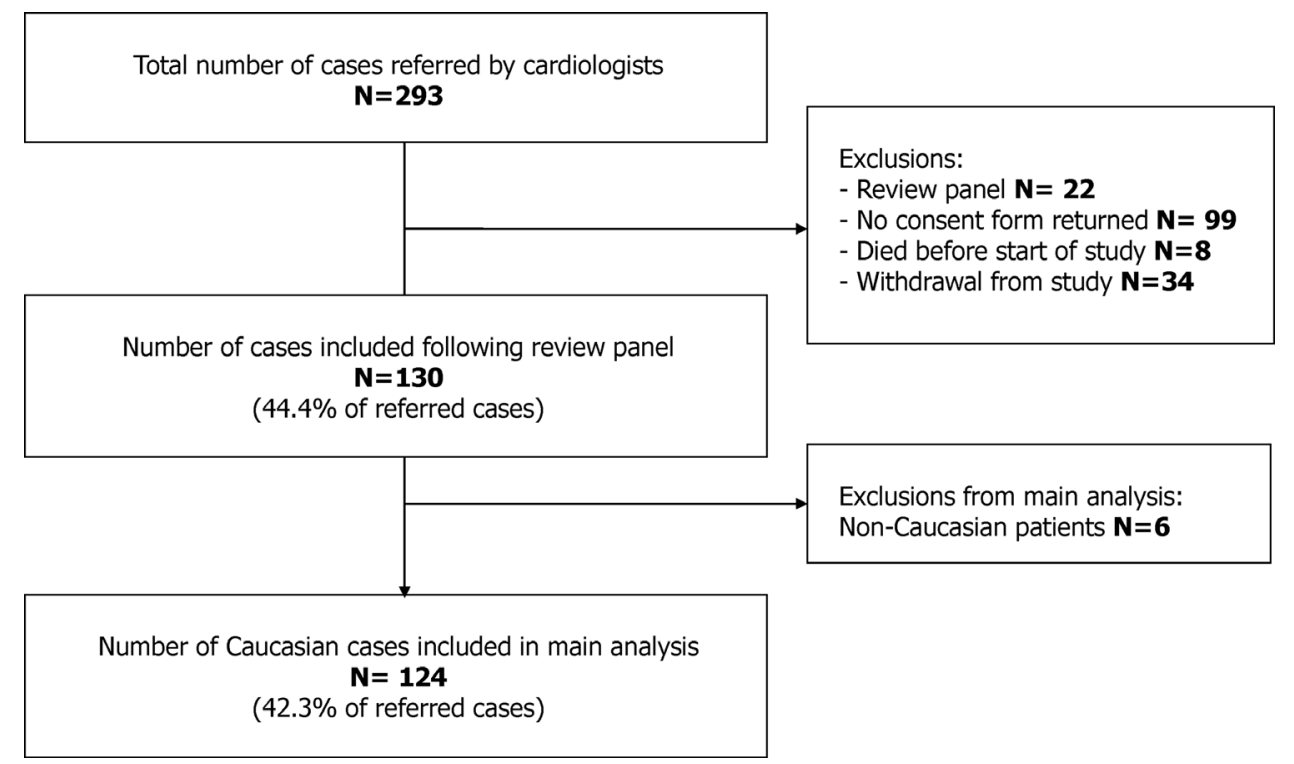

Figure 1 Referrals and cohort accrual.

risk when used according to the label; and conditional risk when there is substantial evidence for QT interval prolongation and TdP risk but only under specific conditions (eg, overdose, interaction with another drug). Drugs were also classified according to cytochrome (CYP) P450 activity (inhibitors and inducers), and potential pharmacodynamics interactions were identified using the Drug Interaction Checker (Medscape). Potential pharmacodynamics interactions were classified into one of the following groups: QTc prolongation; cardiotoxic (non-QTc prolongation-related but other cardiac effect likely, eg, bradycardia or other dysrhythmia); conditional (cardiac effect unclear but drug interaction has an impact

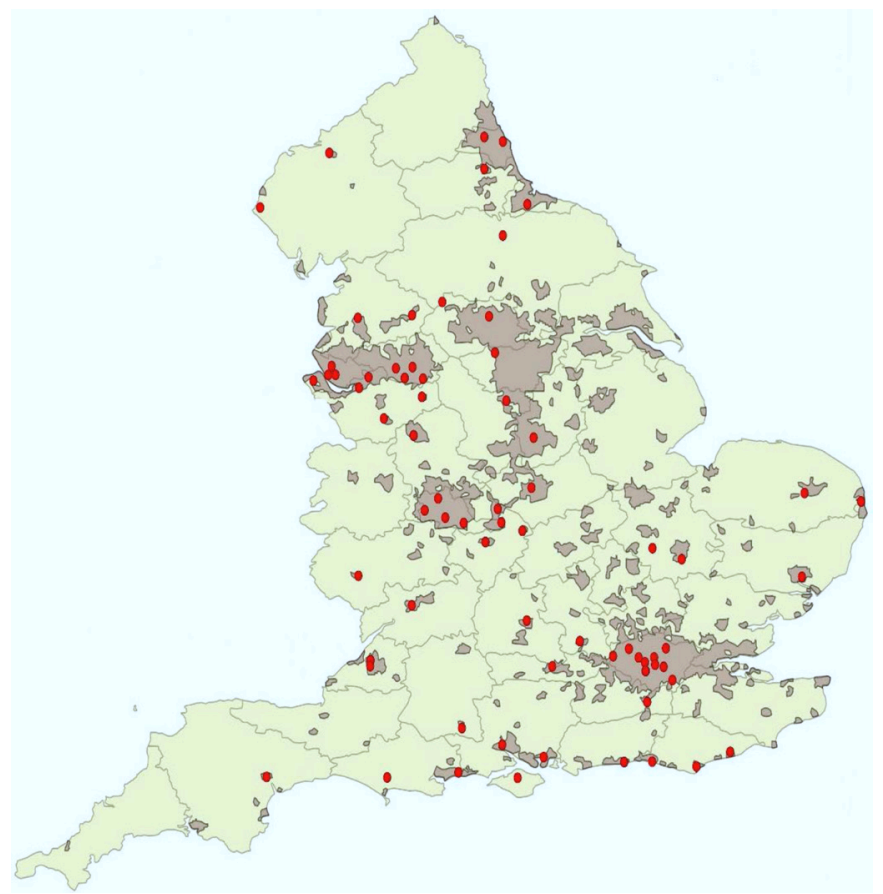

Figure 2 Geographical distribution of consultant cardiologists referring proarrhythmia cases. on a proarrhythmic risk factor, eg, potassium levels); and other (non-cardiovascular) or no drug interaction.

\section{Statistical methods}

Statistical analysis involved descriptive statistics, including measures of central tendency and dispersion for continuous variables (mean, SD, median, range and percentiles) and frequencies with proportions for categorical variables. Results were also stratified according to type of arrhythmia (QT prolongation-associated and/or non-QT prolongation-associated). All statistical analyses were performed using Stata V.12. Radar plots were also constructed in order to characterise cases and estimate the contribution of individual drugs to risk of proarrhythmia. Missing information relating to patients was described using a 'not known' category.

\section{RESULTS}

\section{Case characteristics}

The final overall cohort consisted of 130 cases (figure 1), who were referred from a total of 98 consultant cardiologists across England (figure 2). As the majority of cases were Caucasian $(\mathrm{n}=124,95.4 \%)$, the analysis was performed on these individuals only. Characteristics of the final cohort of Caucasian cases are shown in table 1. Cases were $62.9 \%$ female with a median age at interview of 66 years (IQR 52-73 years). All cases were adults ( $\geq 18$ years). Around a third $(35.5 \%)$ had a family history of sudden death. The types of arrhythmia reported in cases are shown in table 1 . The majority of cases reported $\mathrm{TdP}$, $\mathrm{VF}$ or cardiac arrest with QT prolongation $(\mathrm{n}=79,63.7 \%)$, of whom $56(45.2 \%)$ presented with TdP, $13(10.5 \%)$ presented with VF and $10(8.1 \%)$ presented with TdP and VF. However, 23\% ( $\mathrm{n}=28)$ of cases involved VT or VF not related to QT prolongation. Median (SD) QTc values were 578 (69) $\mathrm{ms}$ in QT prolongation-associated cases and 466.7 (40) ms in non-QT prolongation-associated cases. 


\begin{tabular}{|c|c|c|c|}
\hline Characteristics & $\mathrm{n}$ (\% of cases) & Characteristics & $\mathrm{n}$ (\% of cases) \\
\hline Gender & & QTp associated & 95 (76.6) \\
\hline Female & $78(62.9)$ & TdP, VF, cardiac arrest & $79(63.7)$ \\
\hline Male & $46(37.10)$ & QTp $>500$ ms without symptoms & $9(7.3)$ \\
\hline Age at interview (years) & & QTp ( $\geq 450 \mathrm{~ms}$ in men or $\geq 470 \mathrm{~ms}$ in & \\
\hline $10-19$ & $2(1.6)$ & women) with syncope & $7(5.7)$ \\
\hline 20-29 & $2(1.6)$ & Not associated with QTp & $29(23.4)$ \\
\hline 30-39 & $7(5.7)$ & VTNF & $28(22.6)$ \\
\hline $40-49$ & $15(12.1)$ & Exacerbation of pre-existing VT only & $1(0.8)$ \\
\hline
\end{tabular}

(1)

$\begin{array}{lc}50-59 & 20(16.1) \\ 60-69 & 33(26.6) \\ 70-79 & 37(29.8) \\ 80-89 & 8(6.5) \\ \text { Median (IQR) } & 66(52-73)\end{array}$

\begin{tabular}{lc} 
Smoking status & $13(10.5)$ \\
\hline Current & $58(46.8)$ \\
\hline Ex-smoker & $51(41.1)$ \\
\hline Never smoked & $2(1.6)$ \\
\hline Not known & \\
\hline Alcohol use & $92(74.2)$ \\
\hline Yes & $32(25.8)$ \\
\hline No & \\
\hline Body mass index (kg/m²) & $5(4.0)$ \\
\hline$<18.5$ (underweight) & $55(44.4)$ \\
\hline 18.5-24.9 (normal) & $31(25.0)$ \\
\hline $25-29.9$ (overweight) & $33(26.6)$ \\
\hline F30 (obese) & $17(13.7)$ \\
\hline Family history & $44(35.5)$ \\
\hline Unexplained syncope & \\
\hline Sudden death &
\end{tabular}

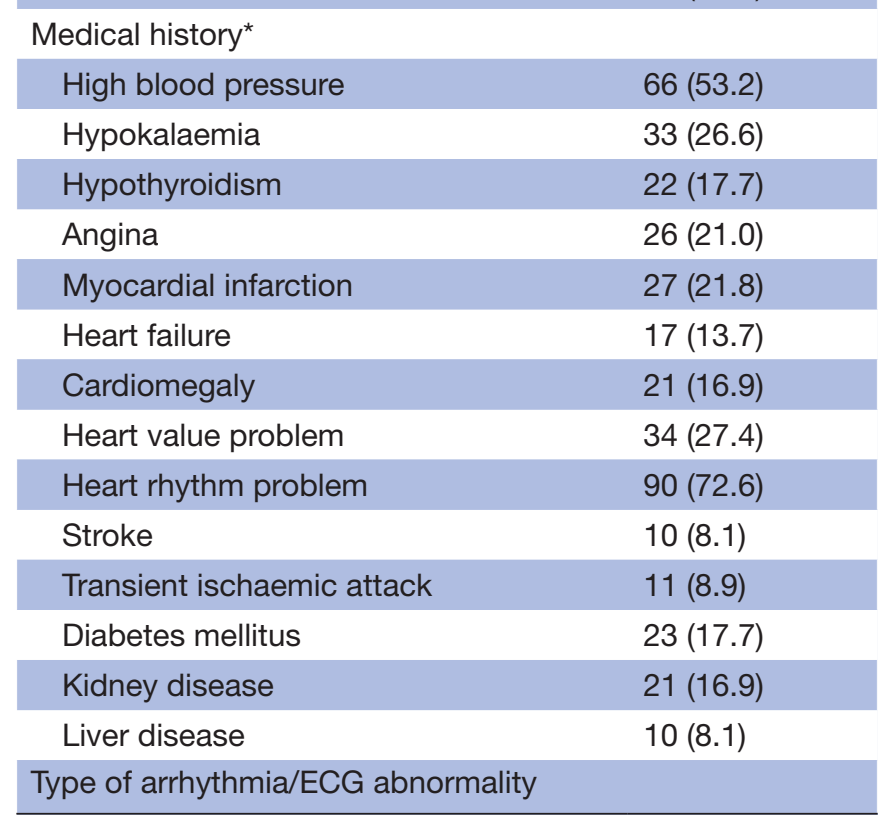

*Self-reported with validation from patient's hospital notes (where available).

QTp, QT interval prolongation; TdP, Torsade de Pointes; VF, ventricular fibrillation; VT, ventricular tachycardia.

\section{Culpable drugs}

A total of 165 patient drug exposures to 42 drugs deemed culpable were identified (table 2, two drugs were unspecified). The most frequently associated drug class was the antiarrhythmics, with 70 drug exposures $(42.4 \%$ of drug exposures) in $67(54.0 \%)$ patients. Amiodarone $(\mathrm{n}=40$; $24.2 \%$ of drug exposures; $32.3 \%$ of patients), flecainide $(\mathrm{n}=23 ; 13.9 \%$ of drug exposures; $18.6 \%$ of patients $)$ and sotalol, a beta-blocker with class III properties $(n=25$; $15.2 \%$ of drug exposures; $20.2 \%$ of patients), were the most frequently reported single drug causes and known to carry a risk of QTc prolongation and/or TdP. Antibiotics (eg, erythromycin; $\mathrm{n}=5,3.0 \%$ of drug exposures; $4.0 \%$ of patients) and antidepressants (eg, citalopram; $\mathrm{n}=7,4.2 \%$ of drug exposures; $5.7 \%$ of patients) were also implicated.

Of the 42 culpable drugs, $14(33.3 \%)$ drugs carried a known risk (120 (72.7\%) drug exposures), 8 (19.0\%) carried a conditional risk (16 (9.7\%) drug exposures), $6(14.3 \%)$ carried a possible risk $(7(4.2 \%)$ drug exposures), 1 (2.4\%) carried a risk in individuals with cLQTS, and $13(31.0 \%)$ carried no known risk of QTc prolongation and/or TdP (18 (10.9\%) drug exposures). The level of risk could not be established for two drugs.

Of the 13 culpable drugs carrying no known risk of QTc prolongation and/or TdP, 2 are known to contribute to bradycardia (timolol and digoxin), 2 are 'not classified' according to CredibleMeds based on the evidence available (cetirizine and verapamil) and 1 remains under active review (lofexidine).

Multiple drug combinations (table 3) were reported in $32(25.8 \%)$ patients. Specifically, $27(21.8 \%)$ patients reported two drugs, $2(1.6 \%)$ patients reported three drugs, $2(1.6 \%)$ patients reported four drugs and $1(0.8 \%)$ patient reported five drugs. Two patients had unspecified drug combinations. Of the patients reporting more than one drug, $84.4 \%$ (27/32) reported using at least one cytochrome P450 inhibitor, with 6 (18.8\%) using two or more in combination. A single patient reported using a CYP inducer. Potential pharmacodynamics interactions within 
Table 2 Drugs culpable in proarrhythmia cases, stratified by risk of QT prolongation and/or Torsades de Pointes

\section{Drug name}

Known risk

Amiodarone

Sotalol

Flecainide

Citalopram

Erythromycin

Clarithromycin

Disopyramide

Domperidone

Fluconazole

Thioridazine

Ciprofloxacin

Haloperidol

Methadone

Pimozide

Possible risk

Venlafaxine

Antihistamine

Capecitabine

Clomipramine

Olanzapine

Tamoxifen

Conditional risk

Furosemide

Amitriptyline

Bendroflumethiazide

Fluoxetine

Amisulpride

Paroxetine

Quinine

Trazodone

Drugs to avoid in CLQTS

Trimethoprim

No known risk

\begin{tabular}{lllll|}
\hline Digoxin* $^{*}$ & C01AA05 & Cardiac glycoside & 4 & 2.4 \\
\hline Propafenone & C01BC03 & Antiarrhythmic & 3 & 1.8 \\
\hline Cetirizine† & R06AE07 & Antihistamine & 1 & 0.6 \\
\hline Chlorpheniramine & R06AB02 & Antihistamine & 1 & 0.6 \\
\hline Dosulepin & N06AA16 & Antidepressant & 1 & 0.6 \\
\hline Lofexidineł & N07BC04 & Drug used in addictive disorders & 1 & 0.6 \\
\hline Loratadine & R06A×13 & Antihistamine & 1 & 0.6 \\
\hline Procaine & S01HA05 & Local anaesthetic & 1 & 0.6 \\
Theophylline & R03DA04 & Drug for obstructive airways disease & 1 & 0.6 \\
\hline Thiazide & C03 & Diuretic & 1 & 0.6 \\
\hline Timoptol & C07AA06 & Beta-blocking agent & 1 & 0.6 \\
\hline & & & & Continued
\end{tabular}

Drug exposures (n) \%
Drug type

\section{Antiarrhythmic}

Beta-blocking agent/antiarrhythmic

Antiarrhythmic

Antidepressant

Antibacterial

Antibacterial

Antiarrhythmic

Propulsive

Antimycotic

Antipsychotic

Antibacterial

Antipsychotic

Drug used in addictive disorders

Antipsychotic

Antidepressant

Antihistamine

Antimetabolite

Antidepressant

Antipsychotic

Antioestrogen

Diuretic

C03CA01

N06AA09

C03AA01

N06AB03

N05AL05

N06AB05

P01BC01

N06A×05

J01EA01

C01AA05

$\mathrm{BCO} 3$

R06AB02

$\mathrm{R} 06 \mathrm{~A} \times 13$

C03

Ant

\section{Antidepressant}

Diuretic

Antidepressant

Antipsychotic

Antidepressant

Antimalarial

Antidepressant

Antibacterial

$\begin{array}{rr}40 & 24.2 \\ 25 & 15.2 \\ 23 & 13.9 \\ 7 & 4.2 \\ 5 & 3.0\end{array}$

4

2.4

2.4

2.4

1.2

1.2

0.6

0.6

0.6

0.6

1

3

1.8

0.6

0.6

0.6

0.6

0.6

3.0

1.8

1.2

1.2

0.6

0.6

0.6

0.6

Continued 
Table 2 Continued

\begin{tabular}{lllrr}
\hline Drug name & ATC code & Drug type & Drug exposures $(\mathbf{n})$ & \% \\
\hline \multicolumn{1}{c}{ Statin } & C10A & Lipid-modifying agent & 1 & 0.6 \\
Verapamil $\dagger$ & C08DA01 & Calcium-channel blocker & 1 & 0.6 \\
Unspecified & - & - & 2 & 1.2 \\
Total & - & - & $\mathbf{1 6 5}$ & $\mathbf{1 0 0 . 0}$ \\
\hline
\end{tabular}

${ }^{*}$ Contributed to bradycardia.

†Not classified-these drugs have been reviewed by CredibleMeds; however, classification could not be performed based on the evidence available and there is no indication the drugs are free of risk of QTp/TdP.

fUnder active review for possible risk of QTp/TdP.

ATC, Anatomical Therapeutic Chemical; cLQTS, congenital long QT syndrome; QTp, QTinterval prolongation; TdP, Torsades de Pointes.

patients reporting more than one drug, according to the Medscape Drug Interaction Checker, were QTc prolongation $(19 / 32,59.4 \%)$, cardiotoxic $(3 / 32,9.4 \%)$, conditional $(1 / 32,3.1 \%)$ and other (non-cardiovascular; $2 / 32$, $3.6 \%)$ interactions. Seven patients $(21.9 \%)$ reported a drug combination without a potential drug interaction.

\section{Types of arrhythmia}

Types of proarrhythmia identified within this study included QT prolongation-related $(\mathrm{n}=95,76.6 \%)$ and non-QT prolongation-related $(\mathrm{n}=29,23.4 \%)$, the latter more typically associated with QRS prolongation (table 2). Stratification according to proarrhythmia type demonstrated few differences between the characteristics of QT prolongation-related and non-QT prolongation-related cases of proarrhythmia, with similar frequency of past medical conditions within both types (figure 3 ). Similarly drugs deemed culpable in cases of proarrhythmia were similar between these types, except for flecainide, which was more commonly implicated with non-QT prolongation-related compared with QT prolongation-related (figure 4).

\section{DISCUSSION}

The DARE study established a cohort of 130 cases of clinically validated drug-induced proarrhythmia referred from across England. To our knowledge this is the largest single study describing a cohort of cases of drug-induced arrhythmia. This information can be used in conjunction with other methods for evaluating the risk of drug-induced arrhythmias, such as spontaneous reports, healthcare databases and active surveillance studies. An analysis of 124 Caucasian cases was undertaken. These Caucasian cases were predominantly female $(62.9 \%)$ and middleaged or elderly (median age at interview of 66 years (IQR 52-73 years)). The majority reported significant past cardiac comorbidity including heart rhythm problems $(72.6 \%)$, high blood pressure $(53.2 \%)$, heart valve problems $(27.4 \%)$, angina $(21.0 \%)$ and myocardial infarction $(21.8 \%)$. This is consistent with data that demonstrate arrhythmia or heart failure to be a risk for proarrhythmia. ${ }^{31}$ Over a third of cases also had an associated family history of sudden death, supporting the potential genetic risk for drug-induced arrhythmia. ${ }^{21}$ Additionally, over a quarter of cases presented with hypokalaemia (26.6\%). Hypokalaemia is associated with QT interval prolongation due to increased competitive blockade of $\mathrm{I}_{\mathrm{Kr}}$, which causes loss of function of the hERG (human Ether-a-go-go Related Gene) channel. ${ }^{16}$

Risk factors for proarrhythmia have been studied before. For example, one study demonstrated hypokalaemia, myocardial infarction, sepsis and heart failure to be risk factors for QT prolongation in both drug-induced and non-drug-induced hospitalised cases. ${ }^{32}$ Furthermore, a study of 21 patients with drug-induced QT prolongation from a Greek hospital found hypertension, female gender, paroxysmal atrial tachyarrhythmias and old age ( $>60$ years) to be common characteristics of patients presenting with the condition. ${ }^{33}$ A study of a group of methadone users in Switzerland demonstrated greater risk of QT prolongation with hypokalaemia, higher methadone dose, altered liver function and use of P450 cytochrome inhibitors. ${ }^{34}$ Finally, a study of psychiatric patients with drug-induced LQTS showed hypokalaemia, abnormal $\mathrm{T}$ wave as well as hepatitis $\mathrm{C}$ and HIV infection to increase the risk of LQTS in this population. ${ }^{35}$

In our study amiodarone, sotalol and flecainide were the most common culpable drugs as was the antiarrhythmic drug group as a whole (42.2\% of drug exposures). Their high prevalence may be due to the relative high potency of cardiac current blockade and/or reflect that cardiologists were the main referral source of cases. For example clinical trials of patients with ventricular and supraventricular arrhythmias treated with sotalol have shown a $4.3 \%$ prevalence of proarrhythmia. ${ }^{36}$ While class Ia, Ic and III antiarrhythmics carry a known risk of QT prolongation and/or TdP and can be potent IKr blockers, amiodarone is often thought of as a rare cause of $\mathrm{TdP} .{ }^{37}$ Its importance in our cohort may be due to its relatively frequent use as an antiarrhythmic agent and/or that those most vulnerable to aLQTS (ie, elderly women with cardiac comorbidity) are more likely to receive amiodarone than other antiarrhythmics due to its perceived lower proarrhythmic risk. This is therefore an important warning to clinicians. Proarrhythmia unrelated to QT prolongation was most commonly observed in users of flecainide, recognised to 


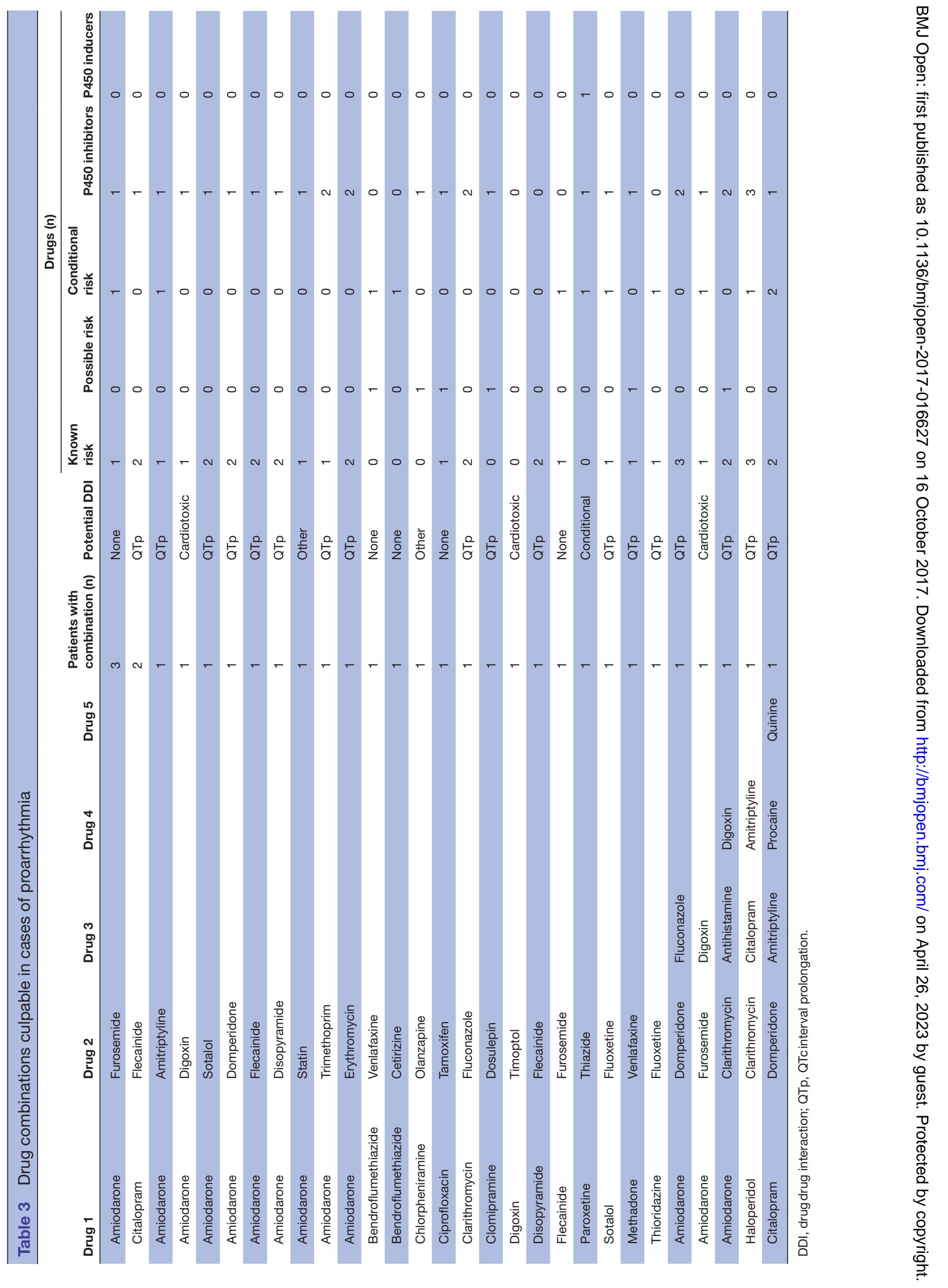




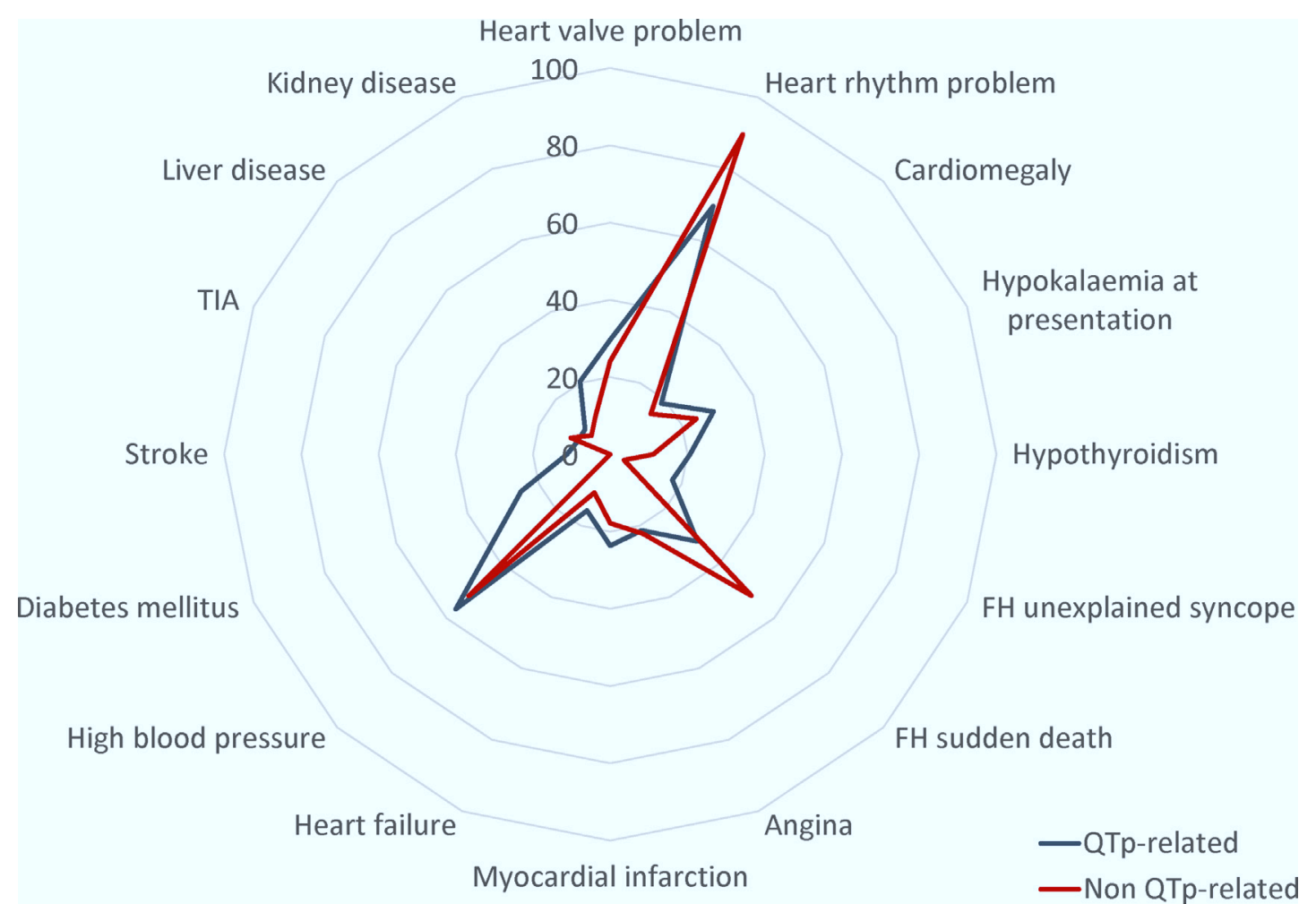

Figure 3 Characteristics of QTp-related and non-QTp-related cases of proarrhythmia. FH, family history; QTp, QTinterval prolongation; TIA, transient ischaemic attack.

result from conduction slowing, causing QRS duration prolongation. ${ }^{38}$ Amiodarone was also frequently reported to be associated with LQTS and TdP in a recent active surveillance study of 58 cases in Germany, a study that showed similar results to DARE, including the identification of hypokalaemia as a risk factor for LQTS and TdP. ${ }^{39}$

Nearly three-quarters of the culpable drug exposures were caused by drugs with known risk of QT prolongation and/ or TdP, including antibiotics and antidepressants. However,
$13(31.0 \%)$ different drugs were diagnosed as culpable in proarrhythmia but not recognised as having such a risk according to the CredibleMeds register. Two are known to contribute to bradycardia (a risk for proarrhythmia), two are 'not classified' according to CredibleMeds based on the evidence available and one remains under active review (lofexidine). Drug combinations were also culpable in a quarter of cases, with up to five drugs being reported in combination. Of these drug combinations by far the

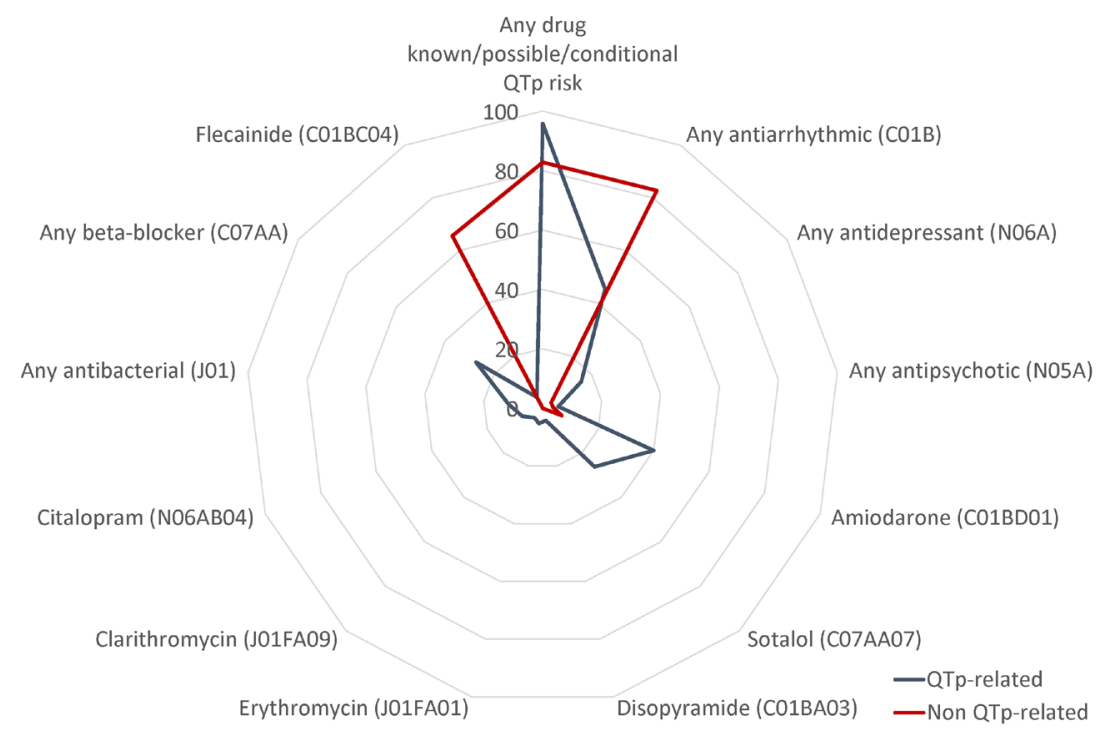

Figure 4 Drugs (Anatomical Therapeutic Chemical codes) culpable in cases of proarrhythmia. QTp, QTinterval prolongation. 
majority, $84.4 \%$ (27/32), included at least one cytochrome P450 inhibitor. Drug combinations of antipsychotics and antidepressants have also previously been shown to increase the risk of QT interval prolongation when compared with antipsychotics alone. ${ }^{40}$ Concurrent use of more than one QT-prolonging drug or concurrent use with a drug that alters the pharmacokinetic profile of the drug is an important risk factor for adverse outcomes. On the other hand, other studies have shown no increased risk of QT interval prolongation with the use of multiple QT-prolonging drug combinations compared with single drugs. ${ }^{32}$ Furthermore, drugs with no known risk of QTc prolongation when used alone may result in prolongation of the QTc interval when used in combination. For example, ceftriaxone and lansoprazole were identified as having a risk of QTc prolongation when used together using electronic healthcare records and in vitro methodology. ${ }^{41}$ Drug interactions were also reported to represent a high proportion of cases of drug-induced $\mathrm{TdP}$ within a Belgium study using the EudraVigilance database (18 of 31 cases). ${ }^{42}$

Limitations of the DARE study include the inability to enrol patients who may have suffered from sudden death as a result of drug-induced arrhythmia; for ethical reasons only live patients could be included. Furthermore, as all referrals came from cardiologists, this may have resulted in selection bias and underestimation of the prevalence of drug-induced arrhythmia from non-cardiac drugs. The level of underestimation due to sudden death or cardiologist referral is difficult to quantify. Determining the cause of death in patients with sudden cardiac death is challenging, with ion channelopathies potentially accounting for $40 \%$ of cases of sudden arrhythmic death syndrome, being undetectable after a patient has died. ${ }^{43}$ A comparison with spontaneous reports of drug-induced arrhythmia might provide information on the level of underestimation of the prevalence of drug-induced arrhythmia from non-cardiac drugs; however, under-reporting of severe adverse drug reactions is known to be high, at approximately $80 \% .{ }^{44}$ In addition, patient answers to questionnaires may have been subject to recall bias and some of the patient information could not be validated from patient records. Finally, differences in ethnicity could not be investigated in this group as there were too few cases of non-Caucasian ethnic origin. Ethnic differences have been shown to affect the risk of QT prolongation due to polymorphisms in cardiac ion channels. ${ }^{45}$ With a larger number of cases, ethnicity differences could be further investigated. It would also be desirable to estimate the incidence of proarrhythmia within the UK population, as has been done for a similar study. ${ }^{39}$ Future work might also involve an investigation of specific drug types and how exposure duration and patterns of usage might affect the risk of proarrhythmias.

\section{CONCLUSIONS}

Increased awareness in the past decade of the public health risk of QT-prolonging drugs has resulted in the regulatory authorities producing guidelines for studying the potential for QT prolongation in premarketing development and the adoption of risk-minimisation measures. ${ }^{29}$ However, due to rarity and diagnostic difficulties, the lack of reported TdP cases in premarketing or postmarketing safety monitoring is a challenge for drug safety. ${ }^{46}$ To date, linked epidemiological and pharmacogenetic data on proarrhythmic events have been lacking. As such, the DARE study has allowed the development of a cohort of cases that provide crucial safety information, as well as underlying clinical and genetic data. ${ }^{18} 4748$ DARE has provided information that confirms risk factors for proarrhythmia, including patient comorbidities and use of drugs with known QTc prolongation risk. However, the study has also identified higher frequency of amiodarone as well as reports of drugs with no known QTc prolongation risk.

Caution is necessary when prescribing class I and III antiarrhythmic drugs. These include amiodarone given its frequent use in clinical practice. The prescriber needs to be aware of a patient's concomitant medications and comorbidities, especially middle-aged to elderly women with cardiovascular disease and/or a family history of sudden death, as well as the likelihood of hypokalaemia. Furthermore prescribers must be aware of the contribution of non-cardiac drugs to the burden of drug-induced arrhythmias, ${ }^{49}$ with approximately $3 \%$ of prescriptions in the UK representing non-cardiac drugs with warnings for arrhythmic potential. ${ }^{50}$ Our findings reinforce the need for safer prescribing of proarrhythmic drugs in clinical practice.

Acknowledgements The authors would like to thank the cardiologists who referred patients to the study, Pauline Telfer for her administrative assistance and Linda Robinson for data collection. The authors also thank the Steering Committee, the British Pacing and Electrophysiology Group (BPEG), now British Heart Rhythm Society (BHRS), and the British Cardiac Society (BCS), which were instrumental in the recruitment of patients. The authors also thank Ed Tong for his statistical support.

Contributors AJC, SAWS, ERB and DL designed the study. VM was responsible for data acquisition. ALC, DL and ERB performed the analyses. ALC, DL, VM, ERB, AJC and SAWS interpreted the findings. ALC, VM, ERB and DL wrote the first draft of the manuscript and revised subsequent versions. The other authors provided input, expertise and critical review of the paper. All authors read and approved the final version of the paper. AJC and SAWS are the guarantors.

Funding The DARE study was funded by the British Heart Foundation under special project grant SP/02/001.

Competing interests ERB was supported by grants from the international Serious Adverse Events Consortium during the conduct of the study. SAWS reports personal fees from ICON, Shire Pharmaceuticals, ONO Pharmaceuticals, Intermune Pharma and IPSEN outside of the submitted work. AJC reports personal fees from Mitsubishi, Laguna, Bayer, Biotronik, Richmond Pharmacology, Boehringer Ingelheim, Daiichi, Menarini, Novartis, St Jude Medical, Bristol-Myers-Squibb, Pfizer, Medtronic, Thrombosis Research Institute, Servier, Boston Scientific, Eli Lilly and Company, and organisational (non-commercial) fees from the European Heart Rhythm Association outside the submitted work.

Ethics approval Ethical approval was gained from the London Multicentre Research Ethics Committee (MREC), reference number MREC/02/2/73.

Provenance and peer review Not commissioned; externally peer reviewed.

Data sharing statement Additional data available on request by emailing abigail. coughtrie@dsru.org. 
Open Access This is an Open Access article distributed in accordance with the Creative Commons Attribution Non Commercial (CC BY-NC 4.0) license, which permits others to distribute, remix, adapt, build upon this work non-commercially, and license their derivative works on different terms, provided the original work is properly cited and the use is non-commercial. See: http://creativecommons.org/ licenses/by-nc/4.0/

(c) Article author(s) (or their employer(s) unless otherwise stated in the text of the article) 2017. All rights reserved. No commercial use is permitted unless otherwise expressly granted.

\section{REFERENCES}

1. Straus SM, Sturkenboom MC, Bleumink GS, et al. Non-cardiac QTCprolonging drugs and the risk of sudden cardiac death. Eur Heart $J$ 2005;26:2007-12.

2. Ben-David J, Zipes DP. Torsades de pointes and proarrhythmia. Lancet 1993;341:1578-82.

3. Brugada P, Wellens HJ. Arrhythmogenesis of antiarrhythmic drugs. Am J Cardiol 1988;61:1108-11.

4. Gonzalez CD, de Sereday M, Sinay I, et al. Endocrine therapies and QTc prolongation. Curr Drug Saf 2010;5:79-84.

5. Bernardi M, Calandra S, Colantoni A, et al. Q-T interval prolongation in cirrhosis: prevalence, relationship with severity, and etiology of the disease and possible pathogenetic factors. Hepatology 1998;27:28-34.

6. Sani MU, Okeahialam BN. QTc interval prolongation in patients with HIV and AIDS. J Natl Med Assoc 2005;97:1657-61.

7. Lazzerini PE, Capecchi PL, Laghi-Pasini F. Long QT syndrome: an emerging role for inflammation and immunity. Front Cardiovasc Med 2015;2:26.

8. Yue $\mathrm{Y}$, Castrichini M, Srivastava $U$, et al. Pathogenesis of the novel autoimmune-associated long-QT syndrome. Circulation 2015;132:230-40.

9. Weissler-Snir A, Gollob MH, Chauhan V, et al. Evaluation of prolonged QT interval: structural heart disease mimicking long QT syndrome. Pacing Clin Electrophysiol 2017:40:417-24.

10. Bellet $\mathrm{S}$. The electrocardiogram in electrolyte imbalance. AMA Arch Intern Med 1955;96:618-38.

11. Takimoto $\mathrm{Y}$, Yoshiuchi $\mathrm{K}$, Kumano $\mathrm{H}$, et al. QT interval and QT dispersion in eating disorders. Psychother Psychosom 2004;73:324-8

12. De Ponti F, Poluzzi E, Cavalli A, et al. Safety of non-antiarrhythmic drugs that prolong the QT interval or induce torsade de pointes: an overview. Drug Saf 2002;25:263-86.

13. Nachimuthu S, Assar MD, Schussler JM. Drug-induced QT interval prolongation: mechanisms and clinical management. Ther Adv Drug Saf 2012;3:241-53.

14. Haverkamp W, Breithardt G, Camm AJ, et al. The potential for QT prolongation and pro-arrhythmia by non-anti-arrhythmic drugs: clinical and regulatory implications. report on a policy conference of the european society of cardiology. Cardiovascular research 2000;47:219-33.

15. Kay GN, Plumb VJ, Arciniegas JG, et al. Torsade de pointes: the long-short initiating sequence and other clinical features: observations in 32 patients. $J$ am coll cardiol 1983;2:806-17.

16. Numaguchi $\mathrm{H}$, Johnson JP, Petersen $\mathrm{Cl}$, et al. A sensitive mechanism for cation modulation of potassium current. Nat Neurosci 2000;3:429-30

17. Sanguinetti MC, Jurkiewicz NK. Two components of cardiac delayed rectifier K+ current. Differential sensitivity to block by class III antiarrhythmic agents. J Gen Physiol 1990;96:195-215.

18. Jamshidi $Y$, Nolte IM, Dalageorgou C, et al. Common variation in the NOS1AP gene is associated with drug-induced QT prolongation and ventricular arrhythmia. J Am Coll Cardiol 2012;60:841-50.

19. Makita N, Horie M, Nakamura T, et al. Drug-induced long-QT syndrome associated with a subclinical SCN5A mutation. Circulation 2002;106:1269-74

20. Paulussen $A D$, Gilissen $R A$, Armstrong $M$, et al. Genetic variations of KCNQ1, KCNH2, SCN5A, KCNE1, and KCNE2 in drug-induced long QT syndrome patients. J Mol Med 2004;82:182-8.

21. Behr ER, Roden D. Drug-induced arrhythmia: pharmacogenomic prescribing? Eur Heart J 2013;34:89-95.

22. Makkar RR, et al. Female gender as a risk factor for torsades de pointes associated with cardiovascular drugs. JAMA 1993;270:2590-7.

23. Yap YG, Camm AJ. Drug induced QT prolongation and torsades de pointes. Heart 2003;89:1363-72.

24. Lehmann MH, Hardy S, Archibald D, et al. Sex difference in risk of torsade de pointes with d, I-sotalol. Circulation 1996:94:2535-41.
25. Khongphatthanayothin A, Lane J, Thomas D, et al. Effects of cisapride on QT interval in children. J Pediatr 1998;133:51-6.

26. Lasser KE, Allen PD, Woolhandler SJ, et al. Timing of new black box warnings and withdrawals for prescription medications. JAMA 2002;287:2215-20.

27. Pratt CM, Hertz RP, Ellis BE, et al. Risk of developing life-threatening ventricular arrhythmia associated with tefenadine in comparison with over-the-counter antihistamines, ibuprofen and clemastine. Am J Cardiol 1994:73:346-52.

28. Wysowski DK, Corken A, Gallo-Torres H, et al. Postmarketing reports of QT prolongation and ventricular arrhythmia in association with cisapride and Food and Drug Administration regulatory actions. Am J Gastroenterol 2001;96:1698-703.

29. ICH. Harmonized Tripartite Guideline E14: The Clinical Evaluation of QT/QTc Interval Prolongation and Proarrhythmic Potential for Nonantiarrhythmic Drugs. 2005.

30. Rock EP, Finkle J, Fingert HJ, et al. Assessing proarrhythmic potential of drugs when optimal studies are infeasible. Am Heart $J$ 2009;157:827-36.

31. Tisdale JE, Patel R, Webb CR, et al. Electro physiologic and proarrhythmic effects of intravenous inotropic agents. Prog Cardiovasc Dis 1995;38:167-80.

32. Tisdale JE, Jaynes HA, Kingery JR, et al. Development and validation of a risk score to predict QT interval prolongation in hospitalized patients. Circ Cardiovasc Qual Outcomes 2013;6:479-87.

33. Letsas KP, Efremidis M, Kounas SP, et al. Clinical characteristics of patients with drug-induced QT interval prolongation and torsade de pointes: identification of risk factors. Clin Res Cardiol 2009;98:208-12

34. Ehret GB, Voide C, Gex-Fabry M, et al. Drug-induced long QT syndrome in injection drug users receiving methadone: high frequency in hospitalized patients and risk factors. Arch Intern Med 2006;166:1280-7.

35. Girardin FR, Gex-Fabry M, Berney P, et al. Drug-induced long QT in adult psychiatric inpatients: the 5-year cross-sectional ECG Screening Outcome in Psychiatry study. Am J Psychiatry 2013:170:1468-76.

36. Soyka LF, Wirtz C, Spangenberg RB. Clinical safety profile of sotalol in patients with arrhythmias. Am J Cardiol 1990;65:74-81.

37. Hohnloser SH, Klingenheben T, Singh BN. Amiodarone-associated proarrhythmic effects. A review with special reference to torsade de pointes tachycardia. Ann Intern Med 1994;121:529-35.

38. Nathan AW, Hellestrand KJ, Bexton RS, et al. The proarrhythmic effects of flecainide. Drugs 1985;29(Suppl 4):45-53.

39. Sarganas G, Garbe E, Klimpel A, et al. Epidemiology of symptomatic drug-induced long QT syndrome and torsade de pointes in germany. Europace 2014;16:101-8.

40. Sala M, Vicentini A, Brambilla P, et al. QT interval prolongation related to psychoactive drug treatment: a comparison of monotherapy versus polytherapy. Ann Gen Psychiatry 2005;4:1.

41. Lorberbaum T, Sampson KJ, Chang JB, et al. Coupling data mining and laboratory experiments to discover drug interactions causing QT prolongation. J Am Coll Cardiol 2016;68:1756-64.

42. Vandael E, Vandenberk B, Vandenberghe J, et al. Cases of druginduced torsade de pointes: a review of belgian cases in the eudravigilance database. Acta Clin Belg 2017:1-6.

43. Behr ER. Inherited heart conditions: Sudden arrhythmic death syndrome. 2009. https://www.bhf.org.uk/-/media/files/publications/ heart-conditions/m111a-life-with-sudden-arrhythmic-deathsyndrome.pdf.

44. Hazell L, Shakir SA. Under-reporting of adverse drug reactions: a systematic review. Drug Saf 2006;29:385-96.

45. Ackerman MJ, Tester DJ, Jones GS, et al. Ethnic differences in cardiac potassium channel variants: implications for genetic susceptibility to sudden cardiac death and genetic testing for congenital long QT syndrome. Mayo clinic proceedings 2003;78:1479-87.

46. Fenichel RR, Malik M, Antzelevitch C, et al. Drug-induced torsades de pointes and implications for drug development. $J$ Cardiovasc Electrophysiol 2004;15:475-95.

47. Behr ER, Ritchie MD, Tanaka T, et al. Genome wide analysis of druginduced torsades de pointes: lack of common variants with large effect sizes. PLoS One 2013;8:e78511.

48. Kaab S, Crawford DC, Sinner MF, et al. A large candidate gene survey identifies the KCNE1 D85N polymorphism as a possible modulator of drug-induced torsades de pointes. Circ Cardiovasc Genet 2012;5:91-9.

49. Yap YG, Camm J. Risk of torsades de pointes with non-cardiac drugs. BMJ 2000;320:1158-9.

50. De Ponti F, Poluzzi E, Montanaro N, et al. QTc and psychotropic drugs. Lancet 2000;356:75-6. 